\title{
Beaucoup de bruit pour ... un microrécit. Lire la littérature courte
}

\author{
Edit Bors* \\ Université Catholique Pázmány Péter, Budapest \\ Institut des Langues Classiques et Néolatines
}

Résumé : Pour susciter l'attention du lecteur, les microrécits intègrent l'implicite et l'allusif, entre autres, dans le ton adopté, dans la structure narrative choisie ou dans l'hybridation générique. Leur réception est aussi liée à des conditions particulières : en peu de temps et avec peu de mots, les microrécits font de l'effet ou passent complètement inaperçus. Certains d'entre eux sont non seulement plaisants à lire mais ils poussent aussi le lecteur à s'interroger sur ses propres habitudes de lecture.

Mots-clés : microrécit, stratégies de lecture, ton, structure narrative, mixité générique

\begin{abstract}
In order to capture their readers' attention, micro-fictions integrate implicit and allusive meanings, among other things, into the adopted tone, the chosen narrative structure or the generic hybridation. Their reception is also determined by limited special conditions: in a short time and in few words, micro-fiction have an effect or remains absolutely imperceptible. Some of the stories are not only enjoyable to read but also push the reader to reflect on his reading skills.
\end{abstract}

Keywords: micro-fiction, reading strategies, tone, narrative structure, generic mixity 
Selon la définition de Laurent Berthiaume, la micronouvelle (le microrécit ou la microfiction) est une fiction littéraire, d'une centaine de mots ou moins, dans laquelle le narrateur doit limiter son texte à l'essentiel, accordant ainsi une place importante au non-dit : "Dans la micronouvelle, le non-dit a préséance sur le dit. On ne lit pas la trame narrative, laquelle est pratiquement absente, mais on l'imagine à son goût personnel, à travers son propre imaginaire » (Berthiaume 2006 : 94). En outre, la microfiction, qui joue de l'implicite et de l'allusif (Montandon 2014), exige de la part du lecteur une participation active, une plus grande attention et imagination, voire une plus grande compétence culturelle. En fait, le lecteur, en vagabondant parmi ces histoires (Montandon 2014) doit adopter une stratégie de lecture toute particulière. La principale caractéristique de ce genre littéraire est de suggérer d'un simple trait l'histoire et les personnages. Comme l'explique Berthiaume :

La micronouvelle m’apparaît en fait à la limite de la nouvelle visible. Plus courte, il n’y a rien. Nous sommes en présence de la nouvelle minimale: quelques mots, moins d'une centaine, pour donner vie à un personnage, dans un lieu, à travers un événement, et une chute pour conclure. (Berthiaume 2006 : 93)

Pour ce faire, la micronouvelle privilégie les images vivantes (idem : 97) et opte pour un ton souvent acide, cynique, côtoyant l'humour noir, le fantastique ou le nonsens.

Ce travail se veut une contribution à l'étude des particularités narrative et générique des microrécits dans une perspective pragmatique et textuelle. Il cherche la réponse à la question de savoir quels mécanismes gèrent le fonctionnement de ces microtextes et comment les compétences de lecture déterminent leur réception. Afin d'explorer le monde de la littérature brève, il portera son attention sur les effets des tonalités, de la structure narrative et de la généricité. En lisant les microrécits, on se rend compte que ces textes courts doivent faire de l'effet en peu de temps et avec peu de mots ou passent complètement inaperçus. Leur réception, avouons-le, est plus ou moins subjective. Tel lecteur, tout en mobilisant ses compétences de lecture, se passionne pour un texte quelconque et passe à côté d'un autre. C'est-ce qui explique aussi le choix subjectif d'un corpus composé de textes plaisants à lire, et qui, à notre sens, incitent le lecteur à déchiffrer leur fonctionnement.

\section{Le jeu des tonalités: l'humour noir, le fantastique et l'absurde}

L'humour noir c'est «la chose triste dite d'un air gai » (Navarri 1998: I164) tel que cela s'observe dans la blague citée par Freud : «le malfaiteur qui est conduit le lundi à la potence émet le propos : ‘Eh bien, la semaine commence bien’ » (Navarri 1998 : 1164). Pour Navarri (1998: 1165), cet exemple témoigne d'un « ajout du plaisir qui vient suspendre l'affect pénible ». En fait, l'humour noir met en scène des thématiques 
socialement tabouées telles que la mort, le suicide ou le handicap. L'incongruité de cette forme d'humour repose sur le fait de rendre comique une manifestation grave, désespérée ou macabre, comme dans l'exemple [ı] :

[1]

\begin{abstract}
[a] Agathe
[b] Toute sa vie, elle était demeurée indécise. [c] Cela créait souvent des situations ambiguës, voire malheureuses, dans lesquelles elle se retrouvait piégée. [d] Fatiguée à force d'hésitations, usée par ses bourdes répétées, elle prit enfin une décision : elle s'enlèverait la vie. [e] D’un pas décidé, sans réfléchir de peur de faire marche arrière, elle se rendit au milieu du pont. [f] Déjà, elle enjambait le garde-fou. [g] Le vent froid de la nuit, les eaux glaciales en contrebas, elle faiblit un instant, mais se reprit aussitôt. [h] Et maintenant, elle tombait en chute libre vers son destin. [i] À mi-parcours, elle changea une dernière fois d'idée... (Berthiaume 2006 : 96-97)
\end{abstract}

Traditionnellement, on distingue trois approches de l'humour qui se complètent éventuellement : la théorie de l'incongruité, la théorie de supériorité et la théorie de soulagement (Nemesi 2018). La première théorie se propose d'expliquer ce qui déclenche l'humour, les deux autres sont centrées sur la fonction psychologique de l'humour. On pourrait alors se demander comment les différents aspects de l'humour se manifestent dans le texte [1] ? On voit bien que cet exemple contient presque tous les éléments relevés par les théories de l'humour. D’un point de vue psychologique, d'après la théorie de supériorité, on rit de la faiblesse de l'héroïne (de son indécision). Selon la théorie de soulagement, le rire permet de nous débarrasser des tensions intérieures ou sociales relatives à la finitude de la vie humaine ou aux conséquences définitives voire mortelles d'une décision. L'incongruité, par contre, vient ici du contraste des traits sémantiques [réversible] vs. [irréversible]. Le sentiment d'humour résulte en effet du mélange inattendu de ces deux traits sémantiques apparemment incompatibles: le [réversible] (changer d’idée) se mêle imperceptiblement à l'[irréversible] (la mort inévitable).

Le fantastique « excelle lorsqu'il est porté par un texte court et le plus souvent par la brièveté de la nouvelle »- dit Prince (2008 : 77). C'est le texte court qui est le plus apte à créer la tension et l'émotion propres aux genres brefs qui se centrent sur peu de personnages, sur un événement unique au caractère inédit et imprévisible et bien sûr, sur l'intensité de l'effet. Le fantastique est aussi le domaine du suggéré, du non-dit, de l'incertitude provoquée entre autres par l'apparition de l'invisible (Prince 2008: 74), comme dans [2] :

[2]

La nuit dans le bloc opératoire 
On avait aspergé le corps d'un liquide qui le rendait fluorescent, et ainsi faisait-on avec chaque organe à mesure qu'on les sortait. L'amusant était que l'on ne pouvait donc opérer que dans le noir. Enfin, l'autopsie confirma que l'homme invisible était bien mort le plus naturellement du monde. (Pastorino $2013: 22$ )

Dans les textes fantastiques, comme le souligne Todorov «L'ambiguïté se maintient jusqu'à la fin de l'aventure : réalité ou rêve ? Vérité ou illusion ? [...] Le fantastique occupe le temps de cette incertitude ; dès qu'on choisit l'une ou l'autre réponse, on quitte le fantastique pour entrer dans un genre voisin, l'étrange ou le merveilleux. Le fantastique, c'est l'hésitation éprouvée par un être qui ne connaît que les lois naturelles, face à un événement en apparence surnaturel ». Dans le texte [2], tout semble être normal: le lieu (« le bloc opératoire »), l'acte («l'autopsie »), le défunt (« le corps ») pourvus d'articles anaphoriques esquissent un contexte connu et habituel (Weinrich 1989). Seule la procédure appliquée (rendre le corps fluorescent et opérer dans le noir) et sa qualification («amusant») signalent l'étrangeté de l'univers où l'invisibilité et la mort naturelle des êtres invisibles sont de règle. Or, c'est justement ce contraste-désigner des phénomènes insolites par les traits sémantiques du [connu]-qui entraîne l'incongruité de l'histoire racontée, et donne au texte une tonalité mixte qu'on pourrait appeler comique de l'absurde.

\section{L'organisation compositionnelle des microrécits}

Comme tout récit, les microrécits doivent se soumettre à certains principes d'organisation compositionnelle. D’après ces principes (Adam 200I : 45-58), ils doivent progresser vers une fin, présenter une succession temporelle, une unité de thème et d'action, obéir aux règles de transformation des prédicats et adopter une mise en intrigue. Une séquence narrative prototypique (idem : 30) est aussi composée d'une Situation initiale (Pnı), Complication (Pn2), Actions (Pn3), Résolution (Pn4), Situation finale (Pn 5) et éventuellement d'une Évaluation finale ou Morale $(\mathrm{Pn} \Omega)$ ayant pour fonction de tirer quelques conclusions des expériences relatées.

Dans l'exemple [I], nous avons affaire à une séquence narrative prototypique (Adam 200I) qui comporte toutes les macropropositions constitutives du récit : Pn I $(a, b), P n 2(c, d), P n 3(e, f, g), P n 4(h, i)$ impliquant Pn5 (la chute ayant pour conséquence la mort plus que probable). Tout compte fait, cet exemple fait plutôt exception. Dans l'ensemble, il nous semble que, contrairement au texte [I] qui n'est qu'un exemple illustratif de sa catégorie, les microrécits étudiés présentent souvent une structure narrative elliptique, comme dans le récit [3] intitulé Godefroy (Berthiaume 2007 : 48) :

[3]

[a] Godefroy 
[b] Clémence fait sa valise. [c] Pour elle tout a été dit. [d] Assis sur le rebord de la fenêtre, Godefroy la supplie du regard. [e] Insensible à son désespoir, elle s'affaire à vider le dernier tiroir de la commode.

[f] Jusqu'à son dernier souffle elle entendra le cri de l'homme dans sa chute. (Marie Ginette Dagenais)

Ce petit texte présente l'intérêt de ne pas respecter la constitution prototypique d'une séquence narrative. Il apparaît clairement qu'un certain nombre de macropropositions sont supprimées, d'où résulte une structure narrative elliptique. On voit bien que la Situation intiale Pnı $(a, b)$ permet d'introduire les deux protagonistes et le cadre de l'histoire, alors que la Complication Pn2 est fournie par (c,d,e). Faute d'explicitation du contexte (absence de dialogues, de descriptions ou de commentaires), c'est grâce à nos savoirs culturels extralinguistiques que nous parvenons à comprendre qu'il est question d'une rupture. Les autres macropropositions (Actions Pn3, Résolution Pn4 et Situation finale Pn5) étant absents ( $\varnothing)$, c'est à partir de l'Évaluation finale $\mathrm{Pn} \Omega(f)$ que nous reconstruisons rétrospectivement la trame du récit. Après une interprétation globale des images, nous nous rendons compte que ce récit retrace l'histoire d'un acte suicidaire: l'homme se jette par la fenêtre (Pn3) et il meurt en conséquence de sa chute (Pn4 et Pn5).

Le microrécit fait donc surtout appel aux connaissances et compétences préalables (culture générale, facultés d’interprétation) du lecteur en guidant son imagination afin qu'il puisse retrouver les composants manquants (Actions Pn3, Résolution Pn4 et Situation finale Pn5) du récit. Dans ces récits minimalistes, "l'inconnu prime sur l'explicite » et « la recherche fait partie de l'acte de lire », dit Berthiaume (2006: 95).

\section{Particularités génériques des microrécits}

Certains microrécits dépassent les catégories génériques habituelles et présentent une mixité inédite ou en rompant tout pacte de lecture, se transforment en un antirécit. Pour expliquer la complexité des genres littéraires, Adam (2014 : 26) propose le terme de généricité qui « permet de penser la participation d'un texte à plusieurs genres ». Cette approche dynamique, que nous adopterons dans notre travail, a l'avantage de pouvoir mettre en théorie toute pratique de mixité générique. Adam (2014 : 26) explique en effet que «Plutôt que de classer les textes dans une catégorie - leur appartenance -, il faut observer les potentialités génériques qui les traversent - leur participation à un ou plusieurs genres ».

La mixité générique peut se manifester par exemple sous forme de reprise et de réécriture d'un texte antérieur dans un autre. Il suffit de citer la notion de transtextualité de Genette (1982), notamment le terme d’intertextualité qui est réservée à la présence effective d'un texte dans un autre. L'intertextualité semble aussi accor- 
der une place primordiale à la réception: elle dépend de la compétence culturelle de chaque lecteur qui peut ne pas percevoir ou, au contraire, projeter le texte antérieur dans le texte lu. L'exemple [4] relève de cette catégorie :

[4]

\section{Trop tard}

Les chiens aboyaient après la caravane. Qui passa néanmoins. C'est regrettable, car elle transportait un virus foudroyant qui (fin du manuscrit)... (Pastorino $2013: 27$ )

Cet exemple est d'autant plus révélateur qu'il illustre un cas de défigement (Bárdosi 200I) et de réécriture du proverbe «Les chiens aboient, la caravane passe ». On voit que la structure figée est disloquée en deux propositions et complétée de nouveaux éléments. Malgré le processus de défigement, on reconnaît aisément, grâce à notre compétence culturelle, l'architexte (Genette 1982), les mots bien connus du texte antérieur et le genre du proverbe. Une citation célèbre (Heinz 2002 : 420), est plus ou moins autoréférentielle : en principe, le lecteur est capable de reconnaître le texte et de lui attribuer un genre. Dans cet extrait, on retrouve aussi des bribes de récit : le proverbe constituerait la Situation initiale Pnı, l'ajout (« elle transportait un virus foudroyant qui ») correspondrait à la Complication Pn2, qui, tout en suscitant une attente provoquée par l'emploi de l'article cataphorique (un virus) et par l'absence de post-information (qui + ø) (Weinrich 1989), clôt promptement le récit. Qui plus est, par allusion au caractère fragmentaire et elliptique de la source, elle semble effacer la possibilité d'une mise en intrigue et d'un dénouement quelconques. En relisant le titre Trop tard, il nous paraît pourtant qu'il s'agit là d'un prolepse (Genette 1972 : 105) qui annonce la fin catastrophique de l'histoire (et de la civilisation ?) ce qui explique, paradoxalement, les lacunes du manuscrit.

En revanche, certains microrécits refusent l’idée même de la généricité en rompant unilatéralement le pacte de lecture (Wagner 2012 : 394) tacite entre l'auteur et le lecteur. Du côté du lecteur, la réception est déterminée par le concept de l'horizon d'attente (Wagner 2012 : 388) qui inclut le partage de certains codes génériques (la règle du jeu), mais aussi « un certain nombre d’idées reçues sur ce qu'est la littérature, certes, mais aussi sur ce qu'est la vie, comme sur le type de relations mimétiques qui peuvent ou non se développer entre ces deux pôles » (Wagner 2012 : 393). Du côté de l'auteur, le pacte de lecture est souple et mobile. Il « autorise transgressions, insuffisance, voire absence du pacte » (Wagner 2012 : 404) pour constituer un espace du jeu. Considérons l'exemple [5] :

[5]

\section{Carnet retrouvé}

(14 mars). Romain m'apprend que maman est morte. (Après-midi). Romain n'est plus 
Romain et maman, évidemment, se porte comme un charme! (16 mars). Ce n'est pas moi qui écris ce journal. (Pastorino 2013 : 29)

On aura vu que le titre du texte [5] demeure ambivalent : d'une part, il fournit au lecteur une indication générique et propose un pacte de lecture spécifique, mais en même temps, la présence même du titre anéantit le genre, comme le dit Lejeune : "Au contact de la fiction, le journal s'étiole, s'évanouit, ou fait une crise d'urticaire »(Lejeune 2007: 10). Pour Lejeune, la rencontre de la fiction et du journal, " c'est une création hybride qui cherche à concilier deux esthétiques contraires" (Lejeune 2007 : 18). Ainsi, le diariste accepte l'absence de la maîtrise d'un avenir imprévisible et incontrôlable contrairement à la «maîtrise imaginaire du romancier» (Lejeune 2007 : 20). L'extrait remplit, de prime abord, certains critères du genre: référence déictique, indication temporelle par les dates, emploi de la première personne, fragmentation, esthétique de la répétition, etc. Toutefois, plus on avance dans la lecture, plus on se heurte à la définition minimale du genre : (a) c'est le diariste qui écrit le journal en se désignant comme je ; (b) le contenu correspond, plus ou moins, à la véracité des événements tels que vécus dans le présent. Malgré cette définition - qui constitue aussi le pacte de lecture -, on voit avec étonnement que dans Le carnet retrouvé, le je dénie même sa propre activité diariste (« ce n'est pas moi qui écris ce journal ») et brouille volontairement la référence des personnes évoquées ("Romain n'est plus Romain »). À cela s'ajoute que le topos du manuscrit retrouvé qui apparaît dans le titre est tellement connoté (Wagner 2012 : 397) que seule sa présence fait preuve de fictionnalité. C'est ainsi qu'on voit le journal, au sein de la fiction, s'auto-détruire.

Venons-en maintenant à l'analyse du texte [6] que nous pouvons considérer comme étant la violation du pacte de lecture :

[6]

Ceci n'est pas un titre

Ça n'a pas débuté comme ça. Ça s'est poursuivi dans une dimension étrangère à toute prévision. Je ne vous dis pas comment ça a fini. (Pastorino 2013 : 19)

Bien que la forme s'adapte, au premier abord, aux critères d'un récit qui porte un titre (Ceci n'est pas un titre), qui a un commencement, un milieu et une fin, ces éléments constitutifs s'évaporent en raison de la négation même de leur fonction narrative. On peut en conclure que ce texte déjoue imprévisiblement l'entente tacite entre l'auteur et le lecteur. Ce dernier, confronté à une micronouvelle, s'attend à lire le récit d'une histoire imaginaire, cependant, au fur et à mesure qu'il avance dans la lecture, il bute sur un antirécit (Wagner 2012 : 289) qui contredit tous les principes de la narrativité. 


\section{Conclusion}

Les microrécits que nous avons analysés, ont ceci de commun que, pour susciter l'attention, ils suprennent le lecteur par un ton narratif tout à fait imprévisible allant de l'humoir noir à l'absurde en passant par le fantastique et le non-sens, qui, malgré la brièveté du genre, sont loin d'être incompatibles. Mais les effets de la tonalité ne suffisent pas à expliquer le fonctionnement des microrécits. Il nous faut aussi prendre en considération la structure narrative elliptique et l'hybridation générique mêlant le récit à d'autres genres notamment au proverbe ou au journal intime. Ces mécanismes fondamentaux exigent un plus grand effort conceptuel de la part du lecteur pour rassembler son imagination, ses savoirs et ses compétences culturelles. Toutefois, la réception des microrécits reste ambiguë : aussi trouve-t-on des récits qui nous laissent indifférents ou, au contraire, nous font rire et pleurer en nous poussant à nous interroger sur des questions générales de la narrativité ainsi que sur nos propres habitudes de lecture.

Note

* Edit Bors est maître de conférences HDR à l'Université Catholique Pázmány Péter de Budapest où elle assure des cours de grammaire descriptive, de stylistique, de linguistique du texte et de l'énonciation. Après des études de philologie romane et de psychologie, elle rédige une thèse de doctorat en sciences du langage portant sur l'approche pragmatique et textuelle de l'autobiographie chez Rousseau, Gide et Sartre. Par la suite, elle consacre sa thèse d’habilitation à l'étude de la langue littéraire dans une perspective contrastive franco-hongroise. Elle est aussi l'auteur de plusieurs articles consacrés à l’analyse des genres littéraires. 


\section{Bibliographie}

Adam, Jean-Michel (2001), Les textes. Types et prototypes, Paris, Nathan.

-- (2014), Genres de récits. Narrativité et généricité des textes, Louvain-la-Neuve, L'Harmattan-Academia.

Bárdosi, Vilmos (200I), « De l'usage à l'usure », Revue d'Études Françaises, 6, pp. 57-6I. Berthiaume, Laurent (2006), «La micronouvelle », Brèves littéraires, 74, pp. 93-98. URL: http://id.erudit.org/iderudit/6044ac (consulté le 16/01/2019).

Berthiaume, Laurent (dir.) (2007), Cent onze micronouvelles, Lorraine, Éditions le Grand Fleuve. URL: http://www.vincentb.sitew.com/QUELQUES LIVRES.G.htm\#QUELQUES_LIVRES.G (consulté le 16/0I/2019).

Genette, Gérard (1972), Figures III, Paris, Seuil.

-- (1982), Palimpsestes : la littérature au second degré, Paris, Seuil.

Heinz, Michaela (2002), "L'exemple lexicographique à fonction culturelle dans le 'Robert pour tous' ", Études de linguistique appliquée, 128, pp. 413-430.

Montandon, Alain (2013), "Formes brèves et microrécits ", Les Cahiers de Framespa

[En ligne] 14, URL: http://journals.openedition.org/framespa/248I(consulté le 29/10/2019)

Lejeune, Philippe (2007), «Le journal comme 'antifiction' », Poétique, 149/1, pp. 3-14.

Navarri, Pascale (1998), "Humour noir, sublimation, quelques questions ", Revue Française de Psychanalyse, 4/12, pp. 1163-1172.

Nemesi, Attila László (2018), « Nyelvészeti humorelméletek », Századvég, 87 ,pp. 41-57. Pastorino, Philippe (2013), Microntes, Sion, Éditions Xenia .URL :http://editions-xenia. $\mathrm{com} / \mathrm{pdf} / \mathrm{doc} / 169$ pastorino-extr.pdf (consulté le 16/0I/2019).

Prince, Nathalie (2008), Le fantastique, Paris, Armand Colin.

Todorov, Tzvetan (1976), Introduction à la littérature fantastique, Paris, Éditions du Seuil.

Wagner, Frank (2012), "Des coups de canif dans le contrat de lecture », Poétique, 172/4, pp. 387-407.

Weinrich, Harald (1989), Grammaire textuelle du français, Paris, Didier/Hatier. 